\title{
ADS-B Mixed sUAS and NAS System Capacity Analysis and DAA Performance
}

\author{
Konstantin J. Matheou (Principal) \\ Zin Technologies Inc. Brook Park, OH, 44142 \\ Rafael D. Apaza, Alan N. Downey, Robert J. Kerczewski, John Wang \\ NASA Glenn Research Center Cleveland, OH, 44135
}

\section{Abstract}

Automatic Dependent Surveillance-Broadcast (ADS-B) technology was introduced more than twenty years ago to improve surveillance within the US National Airspace Space (NAS) as well as in many other countries. Via the NextGen initiative, implementation of ADS-B technology across the US is planned in stages between 2012 and 2025. ADS-B's automatic one second epoch packet transmission exploits on-board GPS-derived navigational information to provide position information, as well as other information including vehicle identification, ground speed, vertical rate and track angle. The purpose of this technology is to improve surveillance data accuracy and provide access to better situational awareness to enable operational benefits such as shorter routes, reduced flight time and fuel burn, and reduced traffic delays, and to allow air traffic controllers to manage aircraft with greater safety margins. Other than the limited amount of information bits per packet that can be sent, ADS-B's other hardlimit limitation is capacity. Small unmanned aircraft systems (sUAS) can utilize limited ADS-B transmission power, in general, thus allowing this technology to be considered for use within a combined NAS and sUAS environment, but the potential number and density of sUAS predicted for future deployment calls into question the ability of ADS-B systems to meet the resulting capacity requirement. Hence, studies to understand potential limitations of ADS-B to fulfill capacity requirements in various sUAS scenarios are of great interest. In this paper we, validate/improve on, previous work performed by the MITRE Corporation concerning sUAS power and capacity in a sUAS and General Aviation (GA) mixed environment. In addition, we implement its inherent media access control layer capacity limitations which was not shown in the MITRE paper. Finally, a simple detect and avoid (DAA) algorithm is implemented to display that ADS-B technology is a viable technology for a mixed NAS/sUAS environment even in proposed larger mixed density environments.

\section{Introduction}

ADS-B modelling and simulation work has been ongoing at NASA's Glenn Research Center (GRC) for the past few years. The motivation to simulate ADS$\mathrm{B}$ technology is due to its acceptance by the Federal Aviation Administration (FAA). Due to the emergence of smaller drones being sold throughout the US and the rapid evolution of drone technology, many safety, commercial, and recreational types of applications will drive the number of drones (aka sUASs) to populate the skies, such that the inclusion of ADS-B technology on future drones may be a logical safety-enhancing extension. Thus, work on two tasks are presented that show simulation results in a mixed sUAS capacity environment, and further extends the analysis to display initial DAA algorithmic results.

\section{Inspiration and Approach}

Thus, the first step is to understand ADS-B performance in a mixed, sUAS and NAS, capacity environment. This has been completed previously by Guterres, Jones, Orrell, and Strain [1]. In work supporting UAS Traffic Management (UTM) research, GRC leveraged the work in [1], validating the results with GRC's ADS-B simulation model. GRC's model includes theoretically proven channel includes theoretically proven channel model algorithms for UTM including: 1) AWGN, 2) link budget, 3) multipath propagation (Fresnel coefficient), and 4) $900-1090 \mathrm{MHz}$ band co-cannel interference, a somewhat different approach from [1]. In implementing individual channel models, the GRC model specific channel impairments to be analyzed, thus allowing better checks to the overall model.

The ADS-B waveform is a Time Division Multiple Access (TDMA) based communications 
modulation [2]. Due to this slotted modulation design, there is an inherent capacity limit at the MAC layer. For air-to-air (A2A) and air-to-ground (A2G) ADS-B communications, there are a total of 3,200 Message Start Opportunity slots (MSOs) [3]. Theoretically the most aerial vehicles (AVs) at one time that can communicate are 3,200. But due to the random way the MSO's are chosen once the link budget is closed, another added layer of throughput interference is inherently added - MSO collisions. This additional functional throughput MSO Collisions algorithm has been added to the GRC ADS-B model. Thus, a more true 'probability of decoding' framed information coming over the air using ADS-B technology can be predicted for high capacity ADS-B usage. This is a performance feature extends the analysis in [1].

From [1], three transceiver types are implemented: 1) ADS-B, 2) Mode S, and 3) Air Traffic Control Radar Beacon System (ATCRBS). All these 3 technology modes share the $900-1090 \mathrm{MHz}$ spectrum, thus the need for co-channel interference algorithm in the GRC model. Also, the GRC model allows for various 'radius ranges' and various heights per 'radius range' that can be altered. The model currently only allows an average constant air speed per AV per 'radius range'. All the above parameters can be altered including transmit power for sUASs. The GRC ADS-B model will be discussed in more detail in the next section.

\section{ADS-B Model Details}

The 'ADS-B Capacity' model was coded for airto-air (A2A) and air-to-ground (A2G) analyses. The simulation was modelled similarly to [1]. The airport is located in the center, bottom of the cylinder at the 3 dimensional point $(0,0,0)$. The 3 dimensions are: 1$)$ distance $x, 2$ ) distance $y$, and 3 ) altitude. The National Air Space (NAS) general aircraft (GAs) are simulated to have an average altitude of $20,000 \mathrm{ft}$. and all have an average speed of $300 \mathrm{~nm} / \mathrm{hr}$. The sUASs, on the other hand, are all randomized in altitude ranging between 50 to $400 \mathrm{ft}$. The sUAS average speed was chosen to be $50 \mathrm{~nm} / \mathrm{hr}$. for all sUASs. All sUASs and GA's initial distance $\mathrm{x}$ and distance $\mathrm{y}$ placement were randomized at the beginning of the simulation to be between 2-21 nm from the center radially. This range was chosen to allow the high density $5 \mathrm{AVs} / \mathrm{km}^{2}$, medium density $3 \mathrm{AVs} / \mathrm{km}^{2}$, and low density 1
$A V / \mathrm{km}^{2}$. Finally, all AV's are incoming/enroute towards the airport radially in a straight line fashion.

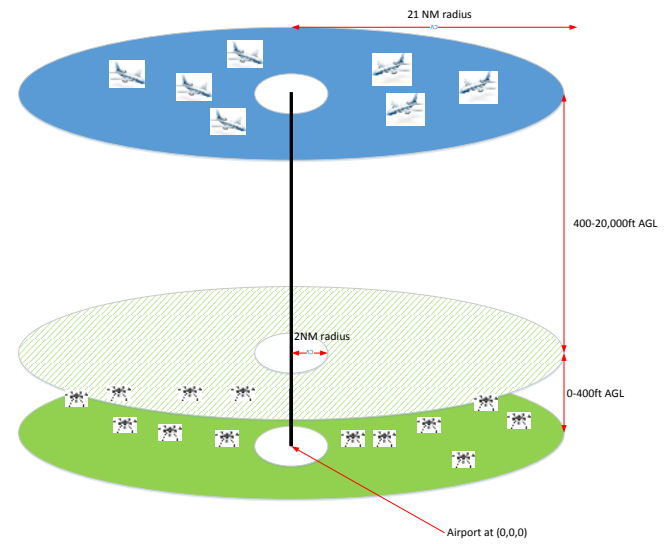

Figure 1- NAS/sUAS Airspace Simulation Approach

It is important to define the types of flying objects referred to in this paper. AV's are the most generalized type of flying objects that include GA and sUASs. GA is the type of aircraft that flies in the NAS, while sUAS are also referred to as drones that is not part of NAS.

In table 3 from [1], there are 16 density scenarios listed. For this paper, scenarios 1 through 12 have been simulated. For traffic density, the AV mix between lower flying sUASs and NAS type flying planes (GA) for all simulations are: $95 \%$ sUAS, 5\% GA, where the types of radar technology for the $5 \%$ GA planes are split as follows: $3 \%$ ADS-B, $1 \%$ Mode $\mathrm{S}$, and $1 \%$ ATCRBS. This mix again was chosen due the approach in [1].

Table 1-MITRE 12 Scenarios

\begin{tabular}{|l|c|c|c|c|c|c|c|}
\cline { 2 - 8 } \multicolumn{1}{c|}{} & \multicolumn{3}{c|}{ Transmit Power (W) } & \multicolumn{3}{c|}{ Traffic Density (AVs/km²) } \\
\cline { 2 - 8 } & $\mathbf{1 . 0 0}$ & $\mathbf{0 . 1 0}$ & $\mathbf{0 . 0 5}$ & $\mathbf{0 . 0 1}$ & $\mathbf{5}$ & $\mathbf{3}$ & $\mathbf{1}$ \\
\hline Scen 1 & $\mathrm{X}$ & & & & $\mathrm{X}$ & & \\
\hline Scen 2 & & $\mathrm{X}$ & & & $\mathrm{X}$ & & \\
\hline Scen 3 & & & $\mathrm{X}$ & & $\mathrm{X}$ & & \\
\hline Scen 4 & & & & $\mathrm{X}$ & $\mathrm{X}$ & & \\
\hline Scen 5 & $\mathrm{X}$ & & & & & $\mathrm{X}$ & \\
\hline Scen 6 & & $\mathrm{X}$ & & & & $\mathrm{X}$ & \\
\hline Scen 7 & & & $\mathrm{X}$ & & & $\mathrm{X}$ & \\
\hline Scen 8 & & & & $\mathrm{X}$ & & $\mathrm{X}$ & \\
\hline Scen 9 & $\mathrm{X}$ & & & & & & $\mathrm{X}$ \\
\hline Scen 10 & & $\mathrm{X}$ & & & & & $\mathrm{X}$ \\
\hline Scen 11 & & & $\mathrm{X}$ & & & & $\mathrm{X}$ \\
\hline Scen 12 & & & & $\mathrm{X}$ & & & $\mathrm{X}$ \\
\hline
\end{tabular}

The basis of this paper's analysis is to understand how the power of sUAS in various high density scenarios affects communications performance in two ways: 1) probability in closing the communications link and 2) capturing a MSO and completing the MAC 
layer process to fully send framed information data to the receiver. Once the signal strength is good enough to enter the ADS-B receiver and there is an available MSO slot in a high ADS-B density scenario, the incoming framed information of the ADS-B signal can be used to begin 'smart' algorithm, one type of which is referred as Detect and Avoid (DAA).

The DAA approach was inspired by [2]. To understand capacity limitations is important, but an initial type of DAA algorithmic analysis should be done to better understand full UTM processing capacity and system performance of ADS-B technology.

\section{DAA Model Details}

Once the framed information passes through the MAC layer (network layer 2), the incoming bit-framed information can be processed. Detect and avoid (DAA) algorithms are processed at higher levels of the network stack. But due to channel impairments, AV ADS-B transceiver capacity, and inherent waveform capacity limitations due to TDMA modulation, the probability of the incoming frame being processed every second epoch will be less than 1.0. As shown in the results sections, the probability of a frame getting through the first time per certain capacity situations can vary from 0.20 to 0.95 . Thus, an analysis using a DAA algorithm may increase the probability to 'track' other adjacent AVs utilizing ADS-B technology. But as always, there is a compromise in other performance parameters that may be lessened. For example, when the detection of a nearby ADS-B transceiver takes longer due to DAA processing, the situation may be too late and a crash may occur.

The DAA approach and design parameter definitions were inherited from [4]. The following DAA design parameter definitions are provided:

1) Measurement Received - means that the link budget of the ADS-B receiver was met and there were no MSO collisions. Thus, the received framed measurement information is then assumed to have been decoded.

2) Set Number - the count of Measurement Received times. Set number minimum is 2 .

3) Track - when a number of Set Number times is counted within a Maximum Size Set.

4) Maximum Set Size - maximum number of measurements that can be missed between two received measurements and allow them to still form a track.

5) Kill Track - the number of times missed MSO slot before stopping to track an AV.

For example, when Max Set Size $=6$, this means a maximum count of 4 MSO slots can be missed between 2 MSO caught slots before a Track is created. When Kill Track $=1$ means that the first missed Measurement Received, the Track will cease to exist and the whole process needs to start over. Using this DAA algorithmic terminology, an analysis of this is done within the next section.

\section{Channel Model Details}

There are 4 algorithmic channel models being implemented within this model: 1) AWGN, 2) Link Budget, 3) Multipath Interference, and 4) Co-channel Interference.

Any communications system is normally baselined using an Average White Gaussian Noise channel. The energy per symbol over noise $(\mathrm{S})$ is used as a parameter within the Link Budget model as shown below equation. For reference, the ADS-B modulation waveform is 8-DPSK. Thus, a total of 3 bits per symbols are sent over the air. Equation 1 sums up the link budget model where, either the minimum symbol power needs to be met, or the maximum transmitter distance can be found within an AWGN channel [3].

$$
R_{\max }=\left(\frac{P_{t} G_{t}^{2} \sigma \lambda^{2}}{\left(4 \pi^{3}\right) S_{\min }}\right)^{1 / 4} \quad \text { eq. } 1
$$

Table 2 is a link budget table example that shows parameters and real values for a link budget. In this particular case an ADS-B transmitter power $\mathrm{Pt}=20 \mathrm{~dB}$ with a certain grazing angle within a smooth surface multipath environment should be able to close the link within $90 \mathrm{~nm}$ (blue and red highlighted values are linear, not $\mathrm{dB}$ ).

Multipath interference model has been duplicated from [1] and [2] using the below equation.

$$
\begin{gathered}
M(E, A)=20 \log \left[C_{o}(E) \exp \left(-2\left(\frac{2 \pi}{\lambda}\right)^{2} s^{2} \sin ^{2}(E)\right)\right]+ \\
g_{T}(E, A)+g_{R}(E, A)
\end{gathered}
$$

Figure 2 is the reproduced Fresnel coefficient value, $\mathrm{C}$, for a smooth surface (worst case) multipath scenario which is the one used in [1]. 
Table 2-Link Budget Example

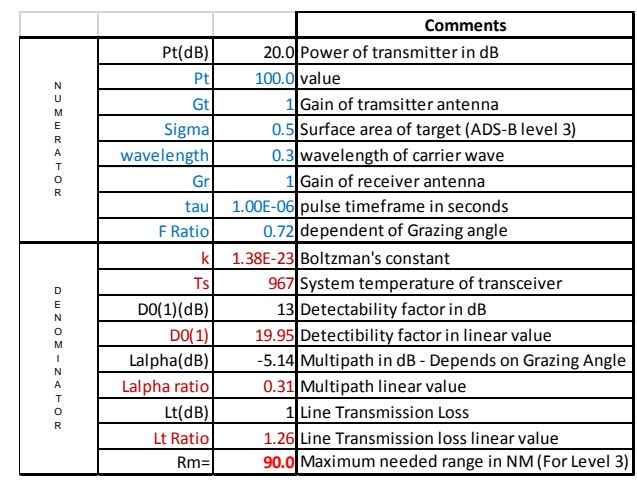

Smooth Surface Reflection Co-efficient

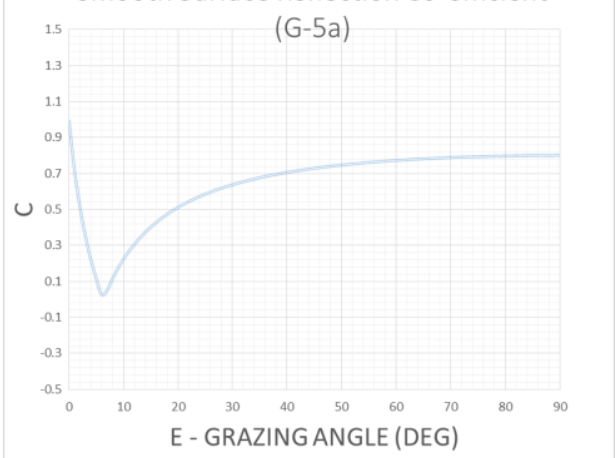

\section{Results and Analysis}

The following sections will present the simulation output and will be contrasted and compared to previous work and then will follow with additional information not presented in previous findings. The UAT system is modelled as an AWGN communication system where additional channel algorithm impairments are used to acquire the probabilistic values for both A2A and A2G implementations. The sections are split by $\mathrm{A} 2 \mathrm{~A}$ and A2G findings.

\section{A. A2A Analysis}

A2A analysis considers the communications between AVs only. In general, there are more multipath affects due to the AV's altitude, speed, and grazing angle. Likewise, depending on AV speed and distance away from each other, the transmission link between AVs may or may not close. The purpose of these simulations is to understand capacity limitations for future mixed sUAS and NAS GA environments. The percentages chosen were to compare to the MITRE previous results. The authors believe these Figure 2 - Fresnel Coefficient Plot for Smooth Surface

ADS-B and the other 2 legacy technologies used currently in the NAS, Mode S, and ATCRBS, utilize the same 980-1090MHz spectrum. [1] implemented a Co-Channel interference model, where the equivalent was implemented with the GRC model. The algorithm output is shown Figure 3.

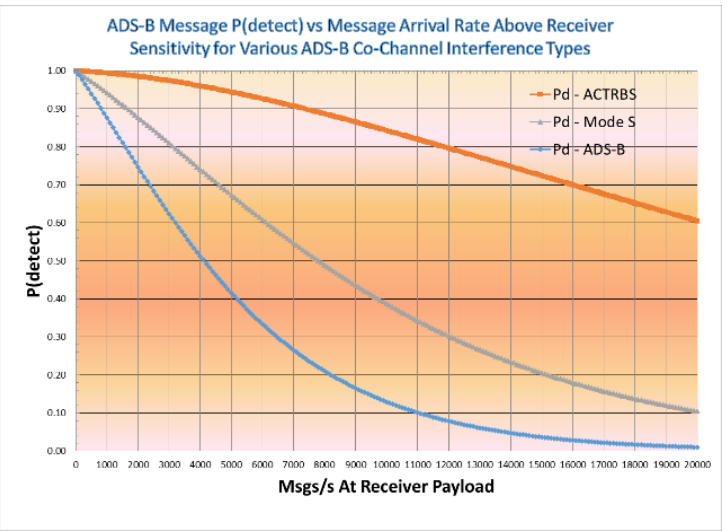

Figure 3 - ADS-B P(detect) vs Message Arrival Rate for Various Co-Channel Interference Types percentages to be different than the ones used, but were kept the same for comparison reasons. Again, the mixed AV environment is a 95\% sUAS using ADS-B UAT, to $3 \%$ GA ADS-B UAT, to $1 \%$ GA Mode S UAT, to $1 \%$ GA ATCRBS. A total of 20,000 AVs for High Density, 12,000 medium Density, and 4,000 AVs for Low Density.

\section{High Density Detailed Analysis}

It was determined a high density environment of $5 \mathrm{AVS} / \mathrm{km}^{2}$ to be implemented with the defined percentage breakdown. sUAS 'communications link' distance was varied while sUAS transmitted power was kept the same for all sUASs. As the distance is varied, the receiving end antenna receiver captures a certain Es/No symbol power (S) level which either closes the link or the link stays open, thus never communicating with the adjacent AV's receiver.

A parameter than was deliberately chosen to be different than [1] was the transmitter power of the GA. The GA ADS-B transmitter power was at $100 \mathrm{~W}$, as opposed to $25 \mathrm{~W}$ that was in [1]. The simulation performance output results in Table 3 show the worst case performance between: 1) 'Close Link Budget' 
which includes co-channel interference, AWGN, and multipath and 2) all channel impairments adding the MSO collisions which is referred to as 'Probability of Decoding'. The values from [1] are in bold.

Table 3 - A2A Worst Case Probabilities

\begin{tabular}{|r|r|r|r|r|}
\cline { 2 - 5 } \multicolumn{1}{c|}{} & \multicolumn{4}{|c|}{ A2A } \\
\cline { 2 - 5 } & \multicolumn{4}{|c|}{ High Density } \\
\hline Scenario & \multicolumn{1}{c|}{$\mathbf{1}$} & \multicolumn{1}{c|}{$\mathbf{3}$} & \multicolumn{1}{c|}{$\mathbf{4}$} \\
\hline Worst Case Prob of Decoding & 0.28 & 0.50 & 0.58 & 0.68 \\
\hline Worst Case Prob of Link Closing & 0.65 & 0.65 & 0.65 & 0.80 \\
\hline From Mitre Table & $<0.25$ & $\mathbf{0 . 1}$ & $\mathbf{0 . 3}$ & $\mathbf{0 . 7 8}$ \\
\hline sUAS Distance MAX & 3.5 & 2.0 & 1.5 & 1.0 \\
\hline & BLOS & BLOS & LOS & LOS \\
\hline
\end{tabular}

We are assuming that the MITRE paper analysis only went as far to 'Probability of Closing Link'. When we add MSO collisions, the probabilities seem to match a little better, but not exactly correlated. It is the opinion of the authors that due to running actual channel algorithms, thus capturing many nuances, our results are more accurate. They also distinguish between the two types of probabilistic performance, 'Probability of Decoding' and 'Probability Closing Link'.

Figure 4 shows the simulation results of the $1.0 \mathrm{~W}$ baseline high density performance output of the GRC simulation. The $\mathrm{x}$ axis shows the 'head-on' distance between sUAS and another sUAS or GA. The power of the sUAS transmitter stays constant, but the 'headon distance' increases. As the distance increases, the probability of a sUAS 'closing the link' starts reducing. This is the black line labelled 'sUAS ABOVE Receiver Operating Point'. Notice the more power, the longer 'Head-On Distance' the sUAS can communicate - see Table 3.

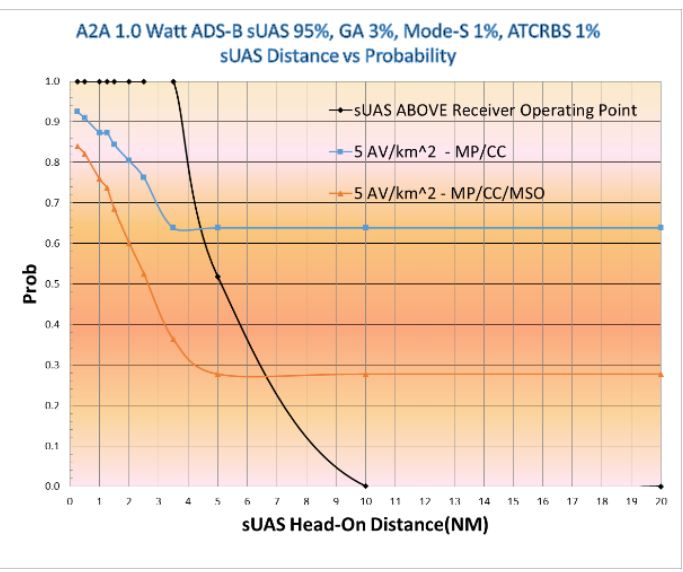

Figure 4 - A2A High Density 1.0W sUAS Transmit Power - Scenario 1
The blue line called ' $\mathrm{MP} / \mathrm{CC}$ ' represents the probability of closing the link when co-channel and multipath channel impairments are added. Finally, the additional MAC layer capacity performance (MSO collisions), once the link is closed after co-channel and multipath, is added. This is the red line called $\mathrm{MP} / \mathrm{CC} / \mathrm{MSO}$ which is the worst case probability of getting an ADS-B frame to the higher network layer levels of the receiver called 'Probability of Decoding'. It is important to note that once the sUAS's head-on distance is too long where the black link budget line is $5-10 \%$ or higher, the probability lines/curves retain their last value. This is because there are no more sUASs to cause more impairments than the last probability value measured.

Figure 5-Figure 7are the remaining High Density scenario plots that map worst case values in Table 3.

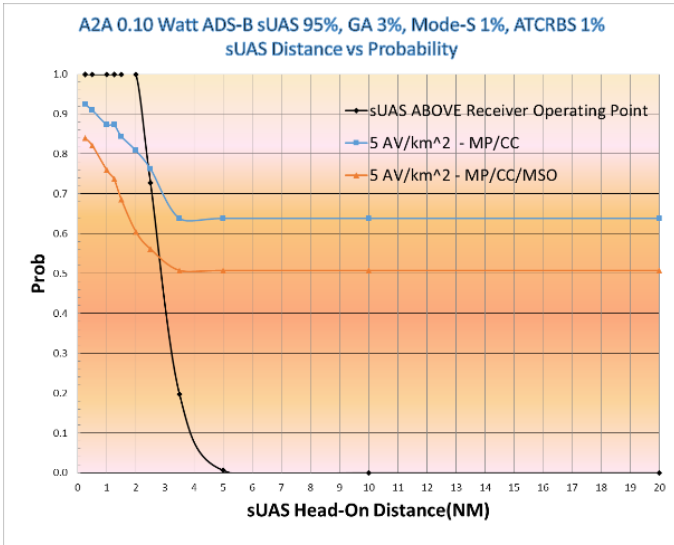

Figure 5 - A2A High Density 0.1W sUAS Transmit Power - Scenario 2

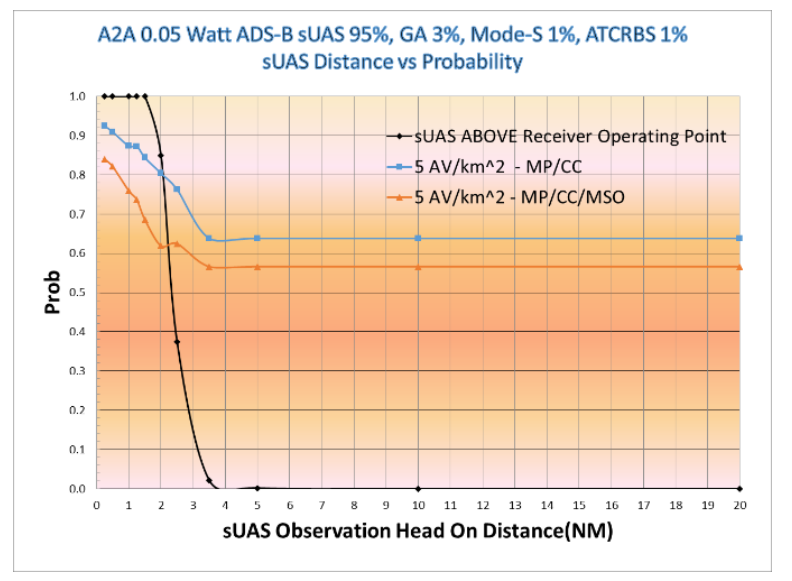

Figure 6 - A2A High Density 0.05W sUAS Transmit Power - Scenario 3 


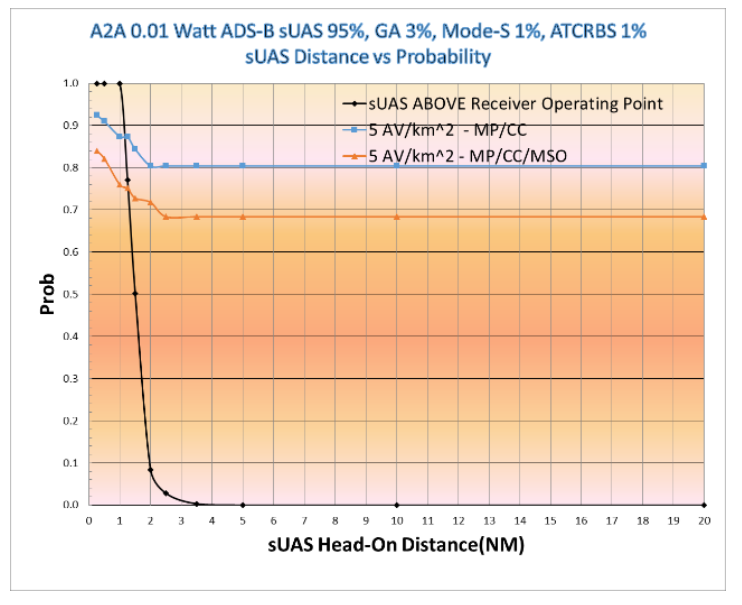

Figure 7 - A2A High Density 0.01W sUAS Transmit Power - Scenario 4

Figure 8 is Scenario 4 from [1]. When you compare the 0.78 'Probability message decode' to the GRC blue line which we assume is equivalent in meaning, they are very similar -0.78 vs 0.80 , but this does not include MSO collisions. When you add the additional MSO collisions probability, the actual 'Probability of Decoding' really is at a worst-case of 0.68 for a high density sUAS environment using $0.01 \mathrm{~W}$ of transmitter power.

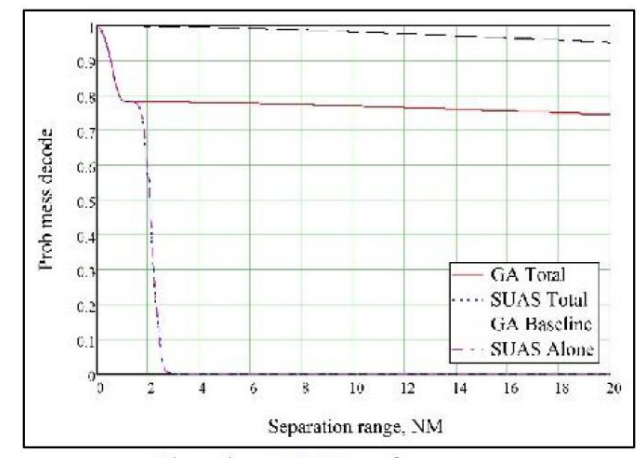

Shared-use UAT performance

\section{Figure 8 - MITRE's A2A High Density 0.01W sUAS Transmit Power - Scenario 4}

The GRC ADS-B model is a Monte-Carlo simulation that uses various channel algorithm models to estimate an Es/No value to close the link. This EsNo value is then compared to the ADS-B receiver operating point of $8 \mathrm{~dB}$ Es/No, which per the standard, is sufficient to meet a BER of 1e-5 [2]. Figure 9shows the tracking of the Es/No values shat show best case and worst case Es/No receiver values. This plot is for Scenario 4.

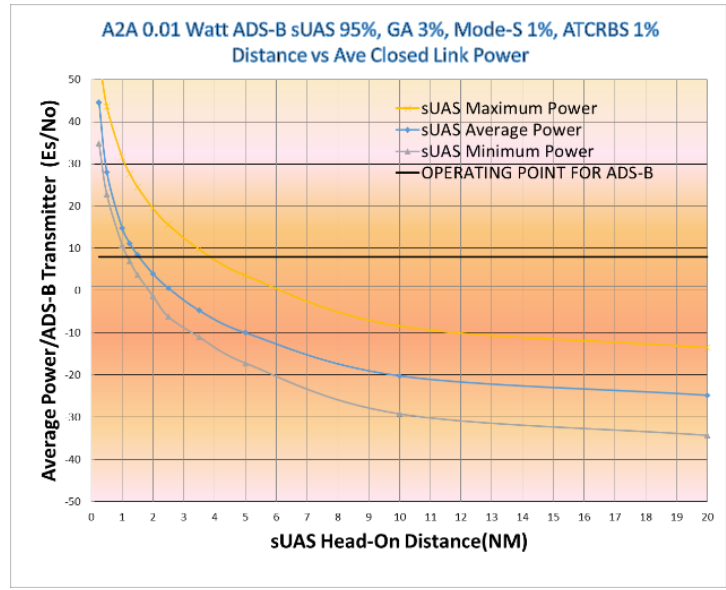

\section{Figure 9 - Scenario 4 Average, Minimum, and Maximum Es/No Levels per sUAS Head-On Distance}

Figure 9 shows, on average, any head-on distance between sUAS and any other type of ADS-B AV that is less than $\sim 1.5 \mathrm{~nm}$ will close the link. To be conservative as what is reflected in the table, the minimum curve is used, thus $1.0 \mathrm{~nm}$ will guarantee the 'closing of the link' $100 \%$ of the time. Of course, we will need to see what the ADS-B MSO collision probability is at this point to ensure that the frame will go through the MAC layer.

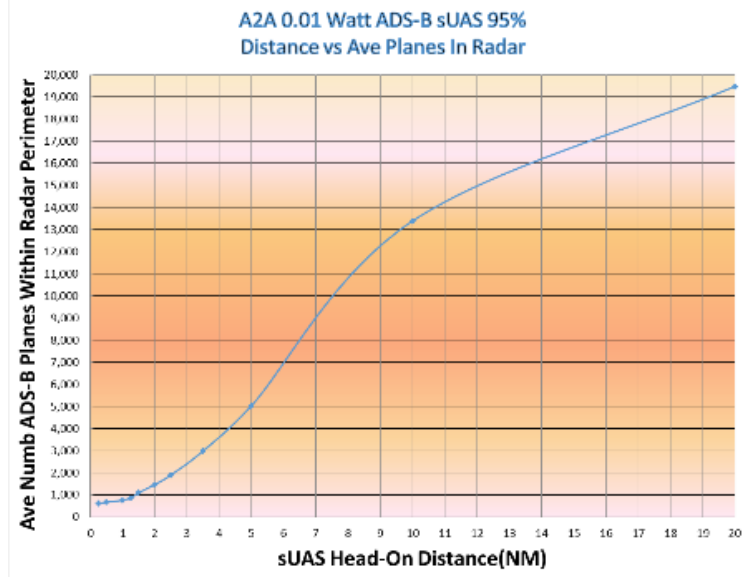

Figure 10 - Scenario 4 Average Number of ADS-B AVs Within Radar Range per sUAS Head-On Distance 
Figure 10 shows how many average number of ADS-B AVs, which includes all sUASs and GAs, which are within each sUAS closing link perimeter.

\section{Medium and Low Density Analysis}

The remaining medium and low density analyses are shown in Table 4. Notice that the GRC simulations results are much more optimistic than those of [1] for 'Probability of Link Closing'.

Table 4 - A2A Worst Case Probabilities for Medium and Low Densities

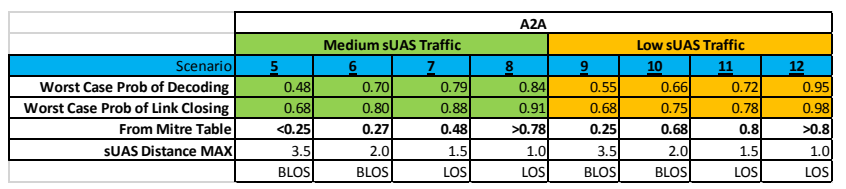

\section{B. A2G Analysis}

The A2G analysis is very similar to the A2A analysis except, the ground station is considered to be always at low altitude, thus the multipath interference will be more constant. See Tables 5and 6.

Table 5 - A2G Worst Case Probabilities for High Density

\begin{tabular}{|r|r|r|r|r|}
\cline { 2 - 5 } \multicolumn{1}{c|}{} & \multicolumn{4}{|c|}{ A2G } \\
\cline { 2 - 5 } & \multicolumn{4}{|c|}{ High sUAS Traffic } \\
\hline Scenario & \multicolumn{1}{c|}{$\underline{\underline{\mathbf{2}}}$} & \multicolumn{1}{c|}{$\underline{\underline{\mathbf{3}}}$} & \multicolumn{1}{c|}{} \\
\hline Worst Case Prob of Decoding & 0.14 & 0.28 & 0.40 & 0.51 \\
\hline Worst Case Prob of Link Closing & 0.18 & 0.33 & 0.52 & 0.60 \\
\hline From Mitre Table & $<.25$ & $<.35$ & $<.1$ & $\mathbf{0 . 3 8}$ \\
\hline sUAS Distance MAX & 3.5 & 2.0 & 1.5 & 1.0 \\
\hline & BLOS & BLOS & LOS & LOS \\
\hline
\end{tabular}

Table 6 - A2G Worst Case Probabilities for Medium and Low Densities

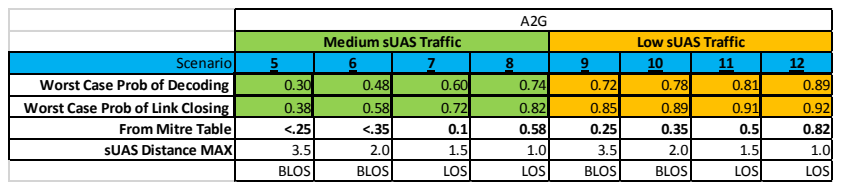

Again, the GRC simulation has a more optimistic worst case probabilities of closing the link.

\section{DAA Analysis}

The following analysis is for DAA algorithm utilizing ADS-B technology. The statistics that are being derived for the Probability to From a Track A2G only. The definitions of the DAA parameters were defined in the above section. The $\mathrm{P}$ (Form a
Track) cannot be captured as a closed form equation, thus simulations are run to capture this DAA statistic.

The first DAA simulation varies the total number of AVs between 100 and 3,000 only utilizing ADS-B technology and is run for a total of 180 seconds, where each ADS-B transmitter will send out its automatic message every second. The 4 defined ADS-B power levels are equally split per ADS-B level categories of $3,2,1$, and sUAS. Thus, if there a total of 1,000 AVs, $250 \mathrm{AVs}$ are dedicated to ADS-B power level 3 which is $250 \mathrm{~W}$. This mix of sUAS to NAS-type GA aerial vehicles, in this task simulation, are $75 \%$ GAs to $25 \%$ sUASs all equally randomized across a $100 \mathrm{NM}$ radius. This is to contrast the previous approach. Due to the larger radar perimeter regions of GA transmitter power levels, most GAs will communicate with the ground station, but not all sUASs will due to their limited $\sim 1 \mathrm{~nm}$ radar perimeter. Again, all AVs are enroute radially to the center where the airport/ground station is placed. For clarity, an example of $1000 \mathrm{AVs}$ parameters are shown in Table 7. Since there are larger powered transmitters in the region, the total number of AVs being detected by the ground station will be close to the total from the beginning of the simulation. Once the simulation begins and the simulation comes close to the $180^{\text {th }}$ second since all $\mathrm{AVs}$ are enroute and radially flying towards the center of the plot, it would be probable that all AVs are being detected by the ground station.

Table 7 - A2G DAA Simulation Input Parameters

\begin{tabular}{|r|r|r|r|r|}
\hline ADS-B Level & Power(dB) & $\begin{array}{c}\text { Amount Randomly Placed } \\
\text { Within 100-5NM Radius }\end{array}$ & AGL(ft) & Speed (NM/hr) \\
\hline 3 & 24 & 250 & 20000 & 300 \\
\hline 2 & 20 & 250 & 20000 & 300 \\
\hline 1 & 14 & 250 & 20000 & 300 \\
\hline sUAS & -20 & 250 & $50-500$ & 50 \\
\hline
\end{tabular}

Table 8 shows the results of the $\mathrm{P}$ (Form a Track) as we adjust both, increasing AVs and increasing MaxSetSize. For example, when MaxSetSize=1, this means that it only takes one Received Message to form a track. We can double-check the situation when AVs=1000 and MaxSizeSet=1 the following way. Since all planes have ADS-B technology, we can refer to the 'co-channel interference' plot and the 'first time MSO collision' plot to validate the $\mathrm{P}$ (Form). From looking at the co-channel interference plot first, $~ 13 \%$ of the AVs do not make it through. Thus, there remain 870 AVs that have to compete for MSOs. The ' $\%$ of First Time MSO Receiver Collisions' for $870 \mathrm{AVs}$ is $\sim 12 \%$. Finally, even though 1,000 AVs are randomly 
placed within the $100 \mathrm{~nm}$ radius, not all AVs will be captured by the ground station, especially since the power of the sUASs is only $0.01 \mathrm{~W}$. So, when taking that small percentage off the total, the $\mathrm{P}$ (Form) matches the simulation's computed output of $\sim 77 \%$. Unfortunately, this double check cannot be done for MaxSetSize $>1$ due to more intense combinational computations. Thus, the reason for a simulation, since a reasonable closed form approach cannot be created.

The simulation results in Table 8 show that as we increase the MaxSetSize variable, the $\mathrm{P}$ (Form) always increases. However, by increasing the MaxSetSize value, the DAA algorithm eventually will not be able to detect the incoming AV as quickly, since we are spending more time to ensure that the probability of forming a track is increased. These are design decisions that will eventually need to be tested and implemented in real flight cases. The purpose of these simulation results is to display the estimated performance of DAA algorithms as we adjust certain parameters.

Table 8 - A2G DAA P(Form) - AVs vs MaxSetSize

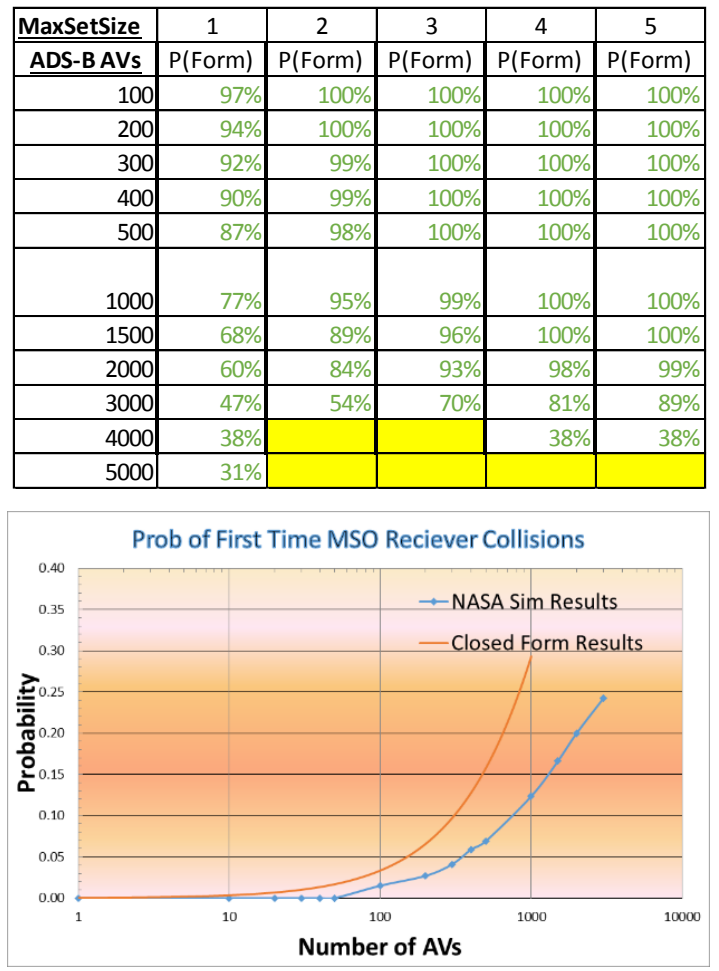

\section{Figure 11 - Probability of First Time MSO Receiver Collisions}

The 'Probability of First Time MSO Receiver Collisions' plot is shown in Figure 11 to display the difference between the estimated closed form equivalent [4] versus the GRC simulation output.

Now we analyze the P(Losing Track). We incorporate the initial step of forming a track, but now we add another DAA parameter called 'Kill Track' where depending on its value will alter the probability of retaining the track. For this analysis, 1,000 ADS-B AVs, all enroute, utilizing the same above simulation parameters. The 1,000 AV amount was chosen because when the DAA parameter MaxSetSize>1, a $\mathrm{P}$ (From) of $95 \%$ will occur. The simulation was run for 180 seconds where an MSO is created per ADS-B per second.

Table 9 - A2G DAA P(Losing Track)

\begin{tabular}{|c|c|c|}
\hline MaxSetSize & measKillTrack & Prob losing Track \\
\hline 2 & 1 & $9.4 \%$ \\
\hline 3 & 1 & $8.8 \%$ \\
\hline 4 & 1 & $8.1 \%$ \\
\hline 5 & 1 & $0.0 \%$ \\
\hline MaxSetSize & measKillTrack & Prob_losing_Track \\
\hline 2 & 2 & $5.1 \%$ \\
\hline 3 & 2 & $4.9 \%$ \\
\hline 4 & 2 & $4.7 \%$ \\
\hline 5 & 2 & $4.5 \%$ \\
\hline MaxSetSize & measKillTrack & Prob_losing_Track \\
\hline 2 & 3 & $3.5 \%$ \\
\hline 3 & 3 & $3.4 \%$ \\
\hline 4 & 3 & $3.3 \%$ \\
\hline 5 & 3 & $3.2 \%$ \\
\hline MaxSetSize & measKillTrack & Prob_losing_Track \\
\hline 2 & 4 & $2.6 \%$ \\
\hline 3 & 4 & $2.5 \%$ \\
\hline 4 & 4 & $2.5 \%$ \\
\hline 5 & 4 & $2.4 \%$ \\
\hline MaxSetSize & measKillTrack & Prob_losing_Track \\
\hline 2 & 5 & $2.1 \%$ \\
\hline 2 & 6 & $1.7 \%$ \\
\hline 2 & 7 & $1.4 \%$ \\
\hline 2 & 8 & $1.2 \%$ \\
\hline 2 & 9 & $1.1 \%$ \\
\hline MaxSetSize & measKillTrack & Prob_losing_Track \\
\hline 3 & 5 & $2.0 \%$ \\
\hline 3 & 6 & $1.7 \%$ \\
\hline 3 & 7 & $1.4 \%$ \\
\hline 3 & 8 & $1.2 \%$ \\
\hline 3 & 9 & $1.1 \%$ \\
\hline
\end{tabular}

As shown in Table 9, increasing the MaxSetSize from 2 to higher values does not affect the 'Probability of Losing Track'. It is very small difference, but it probability needs to be run longer to get the equivalent 
statistical value. We do notice by altering the 'Kill Track' parameter to higher values does affect the Probability of Losing Track.

The next DAA simulation will increase AV capacity. By looking at the previous data, the DAA parameter to close the Track will be held constant at MaxSetSize=2. The DAA parameter 'Kill Track' will be varied to an extreme. Due to higher capacity, simulation time has been reduced to one minute which may affect the statistical soundness.

\section{Table 10 - A2G DAA P(Losing Track) with Increased Capacity}

\begin{tabular}{|r|r|r|r|r|}
\hline ADS-B AVs & MaxSetSize & measKillTrack & TlmeRun(min) & Prob_losing_Track \\
\hline 1500 & 2 & 2 & 3 & $7.2 \%$ \\
\hline 1500 & 2 & 3 & 3 & $5.1 \%$ \\
\hline 1500 & 2 & 4 & 3 & $3.9 \%$ \\
\hline 1500 & 2 & 20 & 3 & $0.6 \%$ \\
\hline ADS-B AVs & MaxSetSize & measKillTrack & TImeRun(min) & Prob_losing_Track \\
\hline 2000 & 2 & 2 & 3 & $9.0 \%$ \\
\hline 2000 & 2 & 5 & 1 & $3.0 \%$ \\
\hline 2000 & 2 & 6 & 1 & $3.1 \%$ \\
\hline 2000 & 2 & 8 & 1 & $2.1 \%$ \\
\hline 2000 & 2 & 20 & 1 & $0.1 \%$ \\
\hline ADS-B AVs & MaxSetSize & measKillTrack & TImeRun(min) & Prob_losing_Track \\
\hline \hline 3000 & 2 & 2 & 1 & $10.7 \%$ \\
\hline 3000 & 2 & 6 & 1 & $4.4 \%$ \\
\hline 3000 & 2 & 20 & 1 & $1.1 \%$ \\
\hline
\end{tabular}

For the highest capacity of AVs run of 3,000, the most feasible parameter setup not to lose tracking is measKillTrack=20, as shown in Table 10. But MaxSetSize must be increased to $>5$ to get to $\mathrm{P}($ Form $)>\% 90$. But again, waiting 20 seconds and depending on speed of each AV, the DAA parameter may be too large for overall safety. A more itemized and critical analysis needs to be done to understand the best sweet spot per capacity amount.

\section{Key Findings}

There are two main tasks that were presented in this paper. The initial task was to simulate scenarios found in [1] concerning capacity in a mixed sUAS and GA environment and to compare results between the two implementations. Added to the first task was further inherent TDMA capacity performance called MSO collisions. Once the mixed sUAS capacity environment was analyzed up to the MAC layer environment, the second task was to begin DAA analysis using a simple algorithm found in [4].

\section{A. Task 1 Key Findings}

- $\quad$ The GRC simulation results - 'Worst Case Probability Closing Link' - do not match with the [1], are much more optimistic for all 3 density cases for both $\mathrm{A} 2 \mathrm{~A}$ and $\mathrm{A} 2 \mathrm{G}$ results

- $\quad$ An $80 \%$ 'Probability to Decode' lower limit has been set by the author to identify worst case performance

- When adding the MSO collisions to the capacity to the simulation, the 'Probability to Decode' is always lower in percentage than the 'Worst Case Probability Closing Link' for both A2A and A2G results

- $68 \%$ 'probability to decode' for the lowest power sUAS transmitter of $0.01 \mathrm{~W}$ in a high density A2A environment is not acceptable

- $\quad 51 \%$ 'probability to decode' for the lowest power sUAS transmitter of $0.01 \mathrm{~W}$ in a high density A2A environment is not acceptable

- $\quad 84 \%$ and $95 \%$ 'probability to decode' for medium and low density A2A environments using the low power $0.01 \mathrm{~W}$ transmitter is a plausible performance findings

- $\quad$ For A2G, only the low density 'probability to decode' for sUAS transmitter power levels of $0.01 \mathrm{~W}$ and $0.05 \mathrm{~W}$ have plausible performance results

- $\quad$ For a mixed sUAS/GA mixed environment due to the low power transmitters are able to meet the $80 \%$ 'probability to decode' cutoff, all sUAS are assumed to be within the Line of Sight (LOS) range $1 \mathrm{NM}$ or less - for both A2A and A2G environments

\section{B. Task 2 Key Findings}

- $\quad$ For $\mathrm{P}($ Form $) \geq 99 \%$ with a capacity of $\sim 1,000$ ADS-B for A2G link, the DAA parameter MaxSetSize $\geq 3$. Thus, it will take 3 seconds to detect an ADS-B nearby transmitter

- $\quad$ For $\mathrm{P}($ Losing the Track $) \leq 1 \%$ with a capacity of $\sim 1,000$ ADS-B for A2G link, the DAA parameter KillTrack $\geq 10$. Thus, it will take 10 seconds for the ADS-B receiver to drop the nearby ADS-B AV 


\section{Conclusion and Future Work}

This paper presented ADS-B modelling that is being done at GRC. The model is constantly being improved from a computational efficiency, to validating its algorithmic results to ensure the probabilities being produced will hopefully closely mimic future real-world high capacity mixed environment scenarios.

As suggested in [1], for others to confirm their results, it is suggested to confirm this paper's results either in a similar algorithmic fashion or in a more efficient, less computational, closed form approach where higher capacity simulations can be found in a quicker timeframe. Now that this work has been published, it would be preferred to collaborate with interested parties to better various to identify the best results.

Due to the algorithmic approach that was taken with the GRC ADS-B capacity model, the results given are with confidence and are more optimistic than the results in [1].

For the DAA algorithmic probability analysis, more work needs to be done to better understand the performance. But at this time, the paper identifies parameter starting points for future real-time on-board DAA processing.

For future work:

1) Incorporate actual NAS and sUAS flight paths and speeds instead of using computer generated AV related data for speed, altitude, and flight path

2) Simulate various sUAS vs GA capacity mixes for A2A DAA simulations

3) Expand the simulation to accept ADS-B frames and extract information to run DAA algorithms with actual ADS-B data

4) Perform DAA A2A analysis similar to the DAA $A 2 G$ analysis in this paper

5) Perform DAA analysis of speed, altitude, and angle using the ADS-B framed information to understand other DAA concepts as described in the DAA paper

\section{References}

[1] Guterres, Michael R., Stanley R. Jones, Gregory

L. Orrell, Robert C. Strain, (January 2017). ADS-B

Surveillance System Performance with Small UAS at Low Altitudes.

[2] RTCA, Inc., (June 2002). Minimum Aviation System Performance Standards for Automatic Dependent Surveillance Broadcast (ADS-B)

[3] Skolnik, Merrill I., (1990). Radar Handbook

[4] Duffoeld, Matthew O., Timothy W. McLain, (January 2017). A Well Clear Recommendation for Small UAS in High-Density, ADS-B Enabled Airspace. 